\title{
Análise da evolução tardia de 291 pacientes submetidos a substituição valvar por próteses metálicas
}

\author{
Carlos M. A. BRANDÃO*, Pablo M. A. POMERANTZEFF*, Luiz C. A. BRANDÃO*, Max GRINBERG*,
} Noedir A. G. STOLF* ${ }^{*}$ Geraldo VERGINELLI*, Adib D. JATENE*

BRANDĀO, C. M. A.; POMERANTZEFF, P. M. A.; BRANDĀO, L. C. A.; GRINBERG, M.; STOLF, N. A. G.; VERGINELLI, G.; JATENE, A. D. - Análise da evolução tardia de 291 pacientes submetidos a substituição valvar por próteses metálicas. Rev. Bras. Cir. Cardiovasc., 10 (1): 50-55, 1995.

RESUMO: No período de janeiro de 1980 a dezembro de 1993, 291 pacientes foram submetidos a substituição valvar por próteses metálicas, no Instituto do Coração do HCFMUSP. Cento e oitenta e sete $(64,3 \%)$ pacientes eram do sexo masculino, com idade variando de 2 meses a 78 anos (média de $38,3+/-18,5$ ). A etiologia das lesões foi reumática em $132(45,4 \%)$ pacientes. Foram realizadas 201 substituiçōes da valva aórtica, 77 da valva mitral, 15 duplas substituiçōes mitro-aórticas, 2 substituiçōes da valva tricúspide, 1 dupla substituição mitro-tricuspídea e 1 tríplice substituição mitro-aórtico-tricuspídea, totalizando 315 substituições valvares. Cirurgias associadas foram realizadas em $164(56,4 \%)$ pacientes, sendo a mais freqũente a correção de aneurisma de aorta ascendente em 49 (16,8\%) pacientes Cento e quarenta e um $(48,4 \%)$ pacientes foram submetidos anteriormente a cirurgias valvares. Os pacientes foram avaliados clinicamente no pós-operatório tardio, segundo a classe funcional (NYHA) e o aparecimento de complicaçōes relacionadas às próteses e à anticoagulação. A mortalidade imediata foi de $36(12,4 \%)$ pacientes. Foram estudados 159 pacientes no pós-operatório tardio, com um tempo médio de evolução de 40,6 meses (10078 meses/paciente). As taxas linearizadas para tromboembolismo, hemorragia relacionada à anticoagulação, óbito tardio, endocardite, escape paravalvar e hemólise no pós-operatório tardio foram, respectivamente, $1,33 \%, 0,95 \%, 1,9 \%, 0,19 \%$, $0,57 \%$ e $0,57 \%$ pacientes/ano. A curva actuarial de sobrevida em 14 anos é de $63,8 \%$. Oitenta e dois por cento dos pacientes encontram-se em classe funcional I no pós-operatório tardio. Podemos concluir que os nossos resultados foram bastante satisfatórios com a utilização de próteses metálicas.

DESCRITORES: Próteses valvulares cardiacas, cirurgia, evolução tardia. Valvas cardíacas, cirurgia.

\section{INTRODUÇÃO}

A qualidade de vida e a sobrevida dos pacientes valvopatas melhora, significativamente, após a cirurgia de substituição valvar. A escolha do substituto valvar depende de uma série de variáveis, as quais devem ser bem avaliadas pelo cirurgião. As próteses metálicas apresentam uma durabilidade maior quando comparadas com as próteses biológicas; no entanto, apresentam riscos significativos de tromboembolismo, e hemorragia relacionada à anticoagulação.

Neste estudo, citamos a experiência da nossa Instituição com as próteses metálicas.

\section{CASUÍSTICA E MÉTODOS}

No período de janeiro de 1980 a dezembro de

Trabalho realizado no Instituto do Coração do Hospital das Clínicas da Faculdade de Medicina da Universidade de São Paulo, São Paulo. SP, Brasil. Apresentado ao $22^{\circ}$ Congresso Nacional de Cirurgia Cardíaca. Brasília, DF, 30 de março a $1^{2}$ de abril, 1995.

*Do Instituto do Coração do Hospital das Clínicas da Faculdade de Medicina da Universidade de São Paulo.

Endereço para correspondência: Carlos Brandão. Av. Dr. Enéas de Carvalho Aguiar, 44. Divisão Cirúrgica. CEP 05403-000 São Paulo, SP, Brasil. Tel.: (011) 898.5432. Fax: (011) 282.2354. 
BRANDÃO, C. M. A.; POMERANTZEFF, P. M. A.; BRANDĀO, L. C. A.; GRINBERG, M.; STOLF, N. A. G.; VERGINELLI, G.; JATENE, A. D. - Análise da evolução tardia de 291 pacientes submetidos a substituição valvar por próteses metálicas. Rev. Bras. Cir. Cardiovasc., 10 (1): 50-55, 1995.

1993, 291 pacientes foram submetidos a substituição valvar por próteses metálicas, no Instituto do Coração do HCFMUSP. Cento e oitenta e sete $(64,3 \%)$ pacientes eram do sexo masculino e 104 $(35,7 \%)$ do sexo feminino. A idade variou de 2 meses a 78 anos, com média de 38,3 anos (desvio padrão de 18,5 anos). A etiologia da valvopatia foi reumática em $132(45,4 \%)$ pacientes, congênita em 21 $(7,2 \%)$, sindrome de Marfan em $16(5,5 \%)$, degenerativa em $11(3,8 \%)$, aneurisma de aorta ascendente em $49(16,8 \%)$, dissecção de aorta ascendente em $32(11 \%)$, endocardite em $7(2,4 \%)$, sifilis em $2(0,7 \%)$ e desconhecida em $21(7,2 \%)$. Os diagnósticos encontrados foram insuficiência mitral em $26(8,9 \%)$ pacientes, estenose mitral em $4(1,4 \%)$, dupla lesão mitral em $4(1,4 \%)$, disfunção de prótese metálica em posição mitral em $4(1,4 \%)$, disfunção de prótese biológica em posição mitral em $55(18,9 \%)$, insuficiência aórtica em $108(37,1 \%)$, estenose aórtica em $25(8,6 \%)$, dupla lesão aórtica em $23(7,9 \%)$, disfunção de prótese metálica em posição aórtica em $7(2,4 \%)$, disfunção de prótese biológica em posição aórtica em $49(16,7 \%)$, insuficiência tricúspide em $3(1 \%)$ e disfunção de prótese metálica em posição tricúspide em $1(0,3 \%)$. Foram encontrados os seguintes diagnósticos associados: aneurisma de aorta ascendente em 49 (16,8\%) pacientes, dissecção de aorta ascendente em 32 $(11 \%)$, insufiçiência coronariana em $11(3,8 \%)$, aneurisma do seio de Valsalva em $3(1 \%)$, escape paravalvar em $3(1 \%)$, abscesso de anel mitral em $1(0,3 \%)$, comunicação interventricular em $4(1,4 \%)$, aneurisma de ventrículo esquerdo em $1(0,3 \%)$ e coarctação de aorta em $1(0,3 \%)$.

Foram realizadas 201 substituições da valva aórtica, 77 da valva mitral, 15 duplas substituições mitro-aórtica, 2 substituiçōes da valva tricúspide, 1 dupla substituição mitro-tricuspídea, e 1 tríplice substituição mitro-aórtico-tricuspídea, totalizando 315

TABELA

TIPOS DE PRÓTESES METÁLICAS UTILIZADAS NO INCOR-HCFMUSP, NO PERIODO DE 1980 A 1993

\begin{tabular}{lc}
\hline TIPO DE PRÓTESE & NÚMERO (TOTAL = 315) \\
\hline Carbomedics & 117 \\
Omnicarbon & 60 \\
St. Jude & 57 \\
Starr-Edwards & 37 \\
Starr-Macchi & 12 \\
Omniscience & 9 \\
Sorin-Bentley & 9 \\
Jatene-Macchi & 4 \\
Björk-Shiley & 3 \\
Lillehei-Kaster & 3 \\
Duromedics & 2 \\
Hall-Kaster & 1 \\
Biocor & 1 \\
\hline
\end{tabular}

TABELA 2

CIRURGIAS ASSOCIADAS À SUBSTITUIÇÃO VALVAR POR PRÓTESES METÁLICAS (TOTAL DE164 PACIENTES).

\begin{tabular}{lc}
\hline CIRURGIAS ASSOCIADAS & NÚMERO (\%) \\
\hline Correção Aneurisma Aorta Ascendente & $49(16,8 \%)$ \\
Correção Disseç̧ão Aorta Ascendente & $32(11 \%)$ \\
Revascularização do Miocárdio & $14(4,8 \%)$ \\
Plástica de DeVega na Tricúspide & $11(3,8 \%)$ \\
Plástica da Valva Mitral & $9(3,1 \%)$ \\
Ventriculoseptoplastia & $4(1,4 \%)$ \\
Correção Aneurisma Seio de Valsalva & $4(1,4 \%)$ \\
Comissurotomia Mitral & $3(1 \%)$ \\
Trombectomia de Átrio Esquerdo & $2(0,7 \%)$ \\
Substituição Mitral Prótese Biológica (PB) & $1(0,3 \%)$ \\
Substituição Tricúspide Prótese Biológica (PB) & $1(0,3 \%)$ \\
Plástica da Valva Aórtica & $1(0,3 \%)$ \\
Comissurotomia Aórtica & $1(0,3 \%)$ \\
Desbastamento da Valva Aórtica & $1(0,3 \%)$ \\
Aneurismectomia de Ventrículo Esquerdo & $1(0,3 \%)$ \\
Ampliação de Anel Aórtico & $1(0,3 \%)$ \\
Tratamento de Abscesso de Anel Aórtico & $1(0,3 \%)$ \\
\hline
\end{tabular}

substituições valvares. Os tipos de próteses metálicas utilizados estão descritos na Tabela 1. Cirurgias associadas foram realizadas em $164(56,4 \%)$ pacientes, sendo a mais freqüente a correção de aneurisma de aorta ascendente (Tabela 2). Cento e quarenta e um $(48,4 \%)$ pacientes foram submetidos a cirurgias valvares anteriores, sendo, na grande maioria, substituições valvares $(81,6 \%$ dos casos).

Quanto à classe funcional (New York Heart Association), $98(33,7 \%)$ pacientes encontravam-se em CF IV no pré-operatório, $114(39,2 \%)$ em CF III, $74(25,4 \%)$ em CF II e $5(1,7 \%)$ em CF I.

Os pacientes foram avaliados clinicamente no pós-operatório tardio segundo a classe funcional (NYHA) e o aparecimento de complicações relacionadas às próteses e à anticoagulação. Todos os pacientes foram submetidos à anticoagulação oral a partir do segundo dia de pós-operatório, com o uso de dicumarínicos.

Para a análise dos resultados foi utilizado o teste exato de Fisher, foram construídas curvas actuariais de sobrevida ${ }^{1}$ pelo método de KaplanMeier (produto-limite), e taxas linearizadas de risco (eventos/,100 pacientes-ano).

\section{RESULTADOS}

Foram acompanhados $159(62,4 \%)$ pacientes no pós-operatório tardio, por um período médio de 40,6 meses. 
BRANDĀO, C. M. A.; POMERANTZEFF,P. M. A.; BRANDĀO, L. C. A.; GRINBERG, M.; STOLF, N. A. G.; VERGINELLI, G.; JATENE,

A. D. - Análise da evolução tardia de 291 pacientes submetidos a substituição valvar por próteses metálicas. Rev. Bras. Cir. Cardiovasc., 10 (1): 50-55, 1995.

TABELA 3

ANÁLISE ESTATISTTICA DA INCIDÊNCIA DE COMPLICAÇŌES PELO TESTE EXATO DE FISHER

\begin{tabular}{lccc}
\hline EVENTO & AÓRTICO $(N=201)$ & MITRAL $(N=77)$ & $p\left({ }^{*}\right)$ \\
\hline Óbito tardio & 9 & 1 & NS \\
Tromboembolismo & 4 & 3 & NS \\
Hemorragia & 3 & 2 & NS \\
\hline
\end{tabular}

(*) probabilidade

A mortalidade imediata foi de $36(12,4 \%)$ pacientes, sendo maior na dupla substituição mitroaórtica (20\%), do que na mitral $(15,6 \%)$ ou na aórtica $(9,4 \%)$. A causa mais freqüente foi o baixo débito cardíaco em $18(50 \%)$ casos. Outras causas de óbito foram sangramento por coagulopatia em 2 $(5,6 \%)$ pacientes, infarto agudo do miocárdio em 2 $(5,6 \%)$ pacientes submetidos a revascularização do miocárdio associadamente, septicemia em 4 (11\%), falência de múltiplos órgãos em $4(11 \%)$, síndrome da angústia respiratória do adulto em 4 (11\%) e acidente vascular cerebral em $2(5,6 \%)$ pacientes.

A mortalidade tardia foi de $10(3,9 \%)$ pacientes, sendo maior na substituição aórtica $(4,9 \%)$ do que na mitral $(1,5 \%)$, apesar de não haver diferença estatisticamente significativa entre os valores ( $\mathrm{Ta}$ bela 3 ). As causas predominantes de óbito tardio foram cardíacas não-valvulares, constituindo $70 \%$ (7) dos casos. Houve 4 óbitos conseqüentes a infarto agudo do miocárdio $(57,1 \%), 2(28,6 \%)$ a arritmia ventricular e $1(14,3 \%)$ a insuficiência cardiaca congestiva. Uma proporção considerável dos óbitos foi de natureza não cardíaca $(30 \%)$.

A sobrevida actuarial foi menor nos pacientes submetidos a substituição aórtica $(62,2 \%)$ do que mitral $(81,3 \%)$. A sobrevida no grupo geral foi de
$63,8 \%$ em 14 anos (Gráfico 1). As taxas de sobrevida actuarial livre de tromboembolismo foram de $84,9 \%$ no grupo geral, $87,0 \%$ e $76,5 \%$ nos pacientes aórticos e mitrais, respectivamente (Gráfico 2). A sobrevida actuarial livre de hemorragia, em 14 anos, foi de $90,4 \%$ no grupo geral e, respectivamente, $89,5 \%$ e $95,6 \%$ no aórtico e mitral (Gráfico 3).

A complicação mais freqüente foi o tromboembolismo, que ocorreu com uma taxa linearizada de $1,33 \%$ pacientes/ano, seguida pela hemorragia relacionada a anticoagulação $(0,95 \%)$, hemólise $(0,57 \%)$, escape paravalvar $(0,57 \%)$ e endocardite $(0,19 \%)$. O tromboembolismo foi mais freqüente no grupo mitral $(2,1 \%)$ do que no aórtico $(1,1 \%)$. $A$ hemorragia também foi mais freqüente no grupo mitral $(1,4 \%)$ do que no aórtico $(0,8 \%)$, apesar de não haver diferença estatisticamente significativa entre os valores (Tabela 3 ). A endocardite teve uma taxa linearizada de $0,26 \%$ no grupo aórtico, e não ocorreu no grupo mitral. O mesmo ocorreu com a hemólise e o escape paravalvar, com taxas linearizadas de $0,8 \%$ no grupo aórtico.

No total, ocorreram 19 complicações relacionadas à prótese (3,61\% pacientes/ano), sendo 14 no grupo aórtico ( $3,76 \%$ pacientes/ano), e 5 no grupo mitral $(3,5 \%$ pacientes/ano).

GRÁFICO 1

CURVAS DE SOBREVIDA

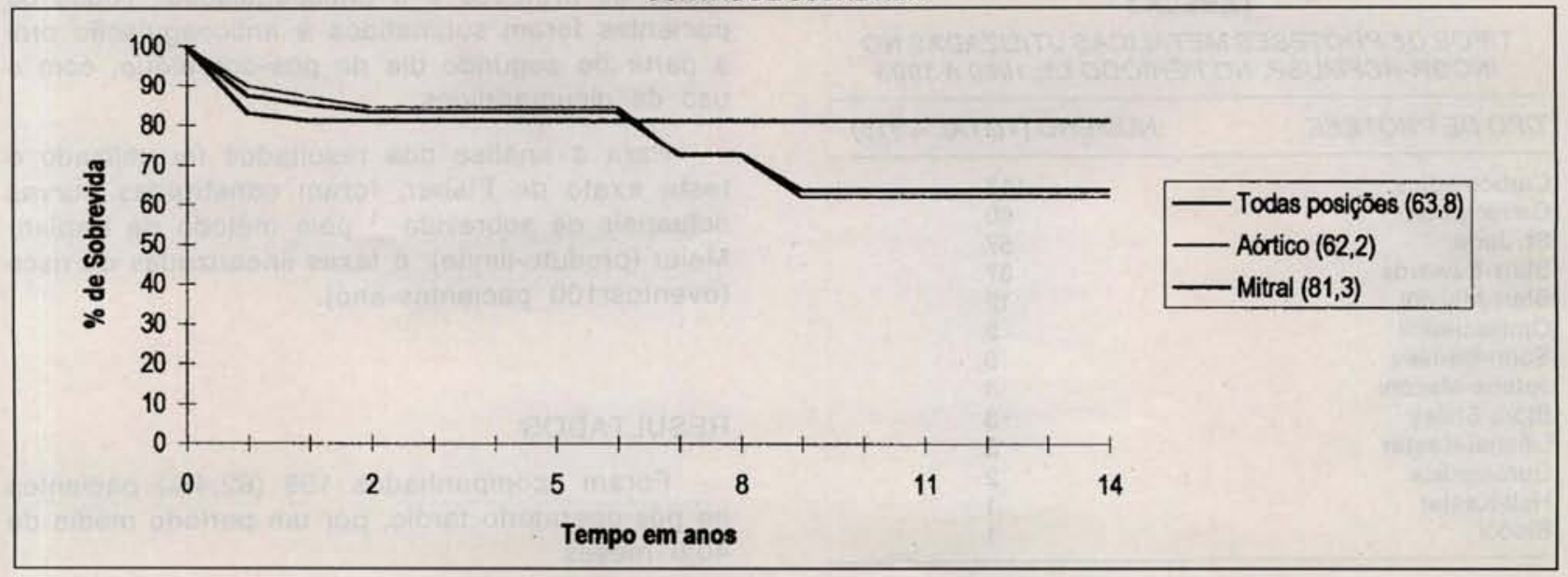


BRANDÃO, C. M. A.; POMERANTZEFF, P. M. A.; BRANDÃO, L. C. A.; GRINBERG, M.; STOLF, N. A. G.; VERGINELLI, G.; JATENE, A. D. - Análise da evolução tardia de 291 pacientes submetidos a substituiçāo valvar por próteses metálicas. Rev. Bras. Cir. Cardiovasc., $10(1): 50-55,1995$.

\section{GRÁFICO 2}

SOBREVIDA LIVRE DE TROMBOEMBOLISMO

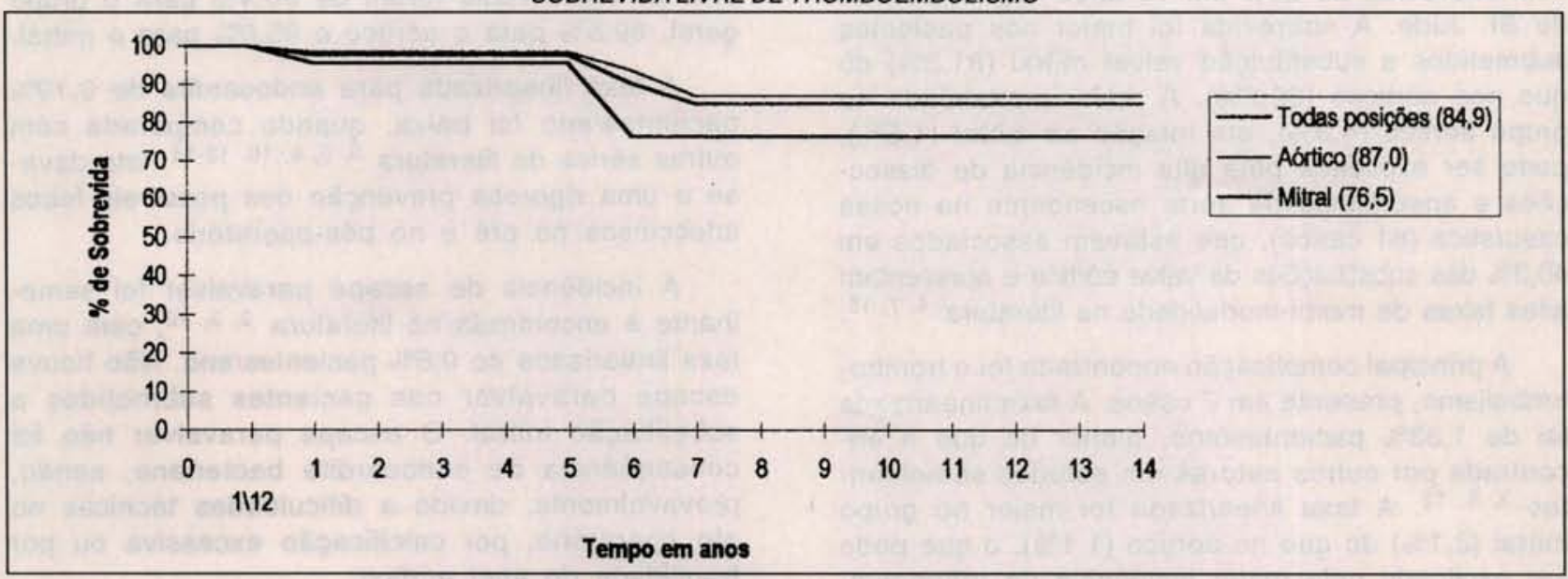

GRÁFICO 3

SOBREVIDA LIVRE DE HEMORRAGIA

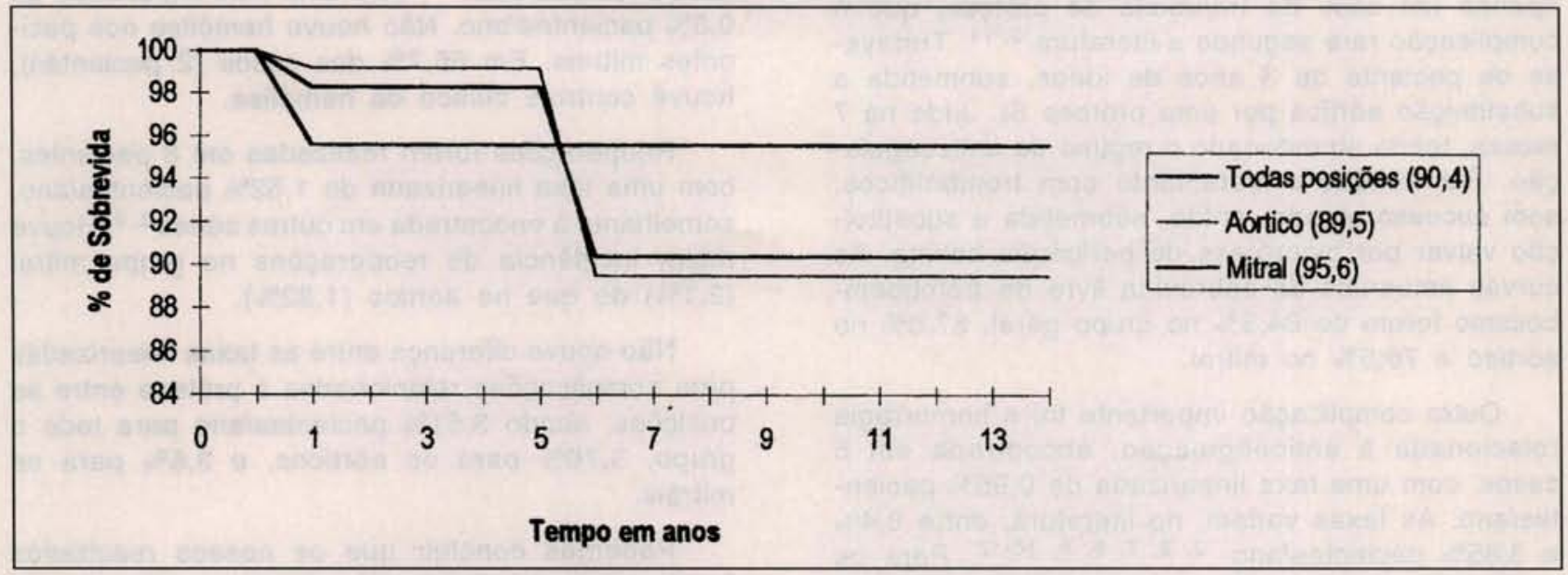

Reoperações ocorreram em 8 pacientes $(1,52 \%$ pacientes/ano), 3 devido a escape paravalvar, 2 devido a disfunção tardia da prótese (estenose), 1 devido a defeito de confecção da prótese (prótese Omniscience com insuficiência), 1 devido a trombose da prótese (paciente abandonou o tratamento com anticoagulante oral) e 1 devido a hemólise importante. As taxas linearizadas nos grupos aórtico e mitral foram $1,33 \%$ ( 5 casos) e $2,16 \%$ pacientes/ ano (3 casos), respectivamente.

Quanto à avaliação clínica, $82,4 \%$ dos pacientes encontravam-se em CF I no pós-operatório tardio, $11,9 \%$ em CF II, $1,9 \%$ em CF III e $3,8 \%$ em CF IV.

Este estudo representa a totalidade da experiência da nossa Instituição com a utilização das próteses metálicas.
A mortalidade hospitalar foi de $12,4 \%$, comparável com outros relatos da literatura 10,11 . Devemos ressaltar que este índice elevado de mortalidade se deve ao fato de que $48,4 \%$ dos casos se tratavam de reoperações e $72,9 \%$ dos pacientes se encontravam em classe funcional III ou IV. Das causas de mortalidade, observamos que $66,7 \%$ foram cardíacas, dentre essas predominantemente - baixo débito cardíaco, concordante com outras séries da literatura $5,8,10$.

As curvas actuariais de sobrevida em 14 anos são bastante favoráveis quando comparadas com outros relatos, como, por exemplo, os de TEPLY et alii ${ }^{15}$, que mostraram uma sobrevida em 10 anos de $56 \%$ com a prótese de Starr-Edwards; CZER et alii ${ }^{5}$, que mostraram sobrevida em 9 anos de $41 \%$, $51 \%$ e $47 \%$ para os pacientes aórticos, mitrais e 
BRANDÃO, C. M. A.; POMERANTZEFF, P. M. A.; BRANDÃO, L. C. A.; GRINBERG, M.; STOLF, N. A. G.; VERGINELLI, G.; JATENE, A. D. - Análise da evolução tardia de 291 pacientes submetidos a substituição valvar por próteses metálicas. Rev. Bras. Cir. Cardiovasc., $10(1): 50-55,1995$.

mitro-aórticos, respectivamente, e NAIR et alii ${ }^{11}$, com sobrevida de $55 \%$ em 10 anos com a prótese de St. Jude. A sobrevida foi maior nos pacientes submetidos a substituição valvar mitral $(81,3 \%)$ do que nos aórticos $(62,2 \%)$. A maior mortalidade no grupo aórtico $(4,9 \%)$, em relação ao mitral $(1,5 \%)$, pode ser explicada pela alta incidência de dissecções e aneurismas de aorta ascendente na nossa casuística ( 81 casos), que estavam associados em $40,3 \%$ das substituições da valva aórtica e apresentam altas taxas de morbi-mortalidade na literatura $4,7,15$.

A principal complicação encontrada foi o tromboembolismo, presente em 7 casos. A taxa linearizada foi de $1,33 \%$ pacientes/ano, menor do que a encontrada por outros autores em estudos semelhantes $3,5,12$. A taxa linearizada foi maior no grupo mitral $(2,1 \%)$ do que no aórtico $(1,1 \%)$, o que pode ser explicado pela maior incidência de tromboembolismo prévio, trombos no átrio esquerdo ou átrio esquerdo volumoso nos pacientes mitrais, que são fatores de risco para a trombogênese 9 . Houve apenas um caso de trombose de prótese, que é complicação rara segundo a literatura 9,14 . Tratavase de paciente de 3 anos de idade, submetida a substituição aórtica por uma prótese St. Jude há 7 meses, tendo abandonado o regime de anticoagulação. Foi tentado o tratamento com trombolíticos, sem sucesso, sendo, então, submetida a substituição valvar por bioprótese de pericárdio bovino. As curvas actuariais de sobrevida livre de tromboembolismo foram de $84,9 \%$ no grupo geral, $87,0 \%$ no aórtico e $76,5 \%$ no mitral.

Outra complicação importante foi a hemorragia relacionada à anticoagulação, encontrada em 5 casos, com uma taxa linearizada de $0,95 \%$ pacientes/ano. As taxas variam, na literatura, entre $0,4 \%$ e $3,85 \%$ pacientes/ano $2,3,5,6,8,10-12$. Para os pacientes aórticos, a taxa foi de $0,8 \%$ pacientes/ ano, enquanto que para os mitrais foi de $1,4 \%$ pacientes/ano. As curvas actuariais de sobrevida livre $d$ s hemorragia foram de $90,4 \%$ para o grupo geral, $89,5 \%$ para o aórtico e $95,6 \%$ para o mitral.

A taxa linearizada para endocardite de $0,19 \%$ pacientes/ano foi baixa, quando comparada com outras séries da literatura $3,5,8,10,12-14$. Isto devese a uma rigorosa prevenção dos possíveis focos infecciosos no pré e no pós-operatório.

A incidência de escape paravalvar foi semeIhante à encontrada na literatura $2,3,10$, com uma taxa linearizada de $0,8 \%$ pacientes/ano. Não houve escape paravalvar nos pacientes submetidos a substituição mitral. O escape paravalvar não foi conseqüência de endocardite bacteriana, sendo, provavelmente, devido a dificuldades técnicas no ato operatório, por calcificação excessiva ou por fragilidade do anel aórtico.

A ocorrência de hemólise, não relacionada a escape paravalvar, foi maior do que a encontrada em outros estudos 3,5 , com uma taxa linearizada de $0,8 \%$ pacientes/ano. Não houve hemólise nos pacientes mitrais. Em $66,7 \%$ dos casos (2 pacientes), houve controle clínico da hemólise.

Reoperações foram realizadas em 8 pacientes, com uma taxa linearizada de $1,52 \%$ pacientes/ano, semelhante à encontrada em outras séries ${ }^{2,3}$. Houve maior incidência de reoperações no grupo mitral $(2,1 \%)$ do que no aórtico $(1,32 \%)$.

Não houve diferença entre as taxas linearizadas para complicações relacionadas à prótese entre as posições, sendo $3,61 \%$ pacientes/ano para todo o grupo, $3,76 \%$ para os aórticos, e $3,5 \%$ para os mitrais.

Podemos concluir que os nossos resultados foram bastante satisfatórios com a utilização de próteses metálicas, independente da posição. 
BRANDÃO, C. M. A.; POMERANTZEFF, P. M. A.; BRANDÃO, L. C. A.; GRINBERG, M.; STOLF, N. A. G.; VERGINELLI, G.; JATENE, A. D. - Análise da evolução tardia de 291 pacientes submetidos a substituição valvar por próteses metálicas. Rev. Bras. Cir. Cardiovasc., 10 (1): 50-55, 1995.

RBCCV 44205-258

BRANDĀO, C. M. A.; POMERANTZEFF, P. M. A.; BRANDĀO, L. C. A.; GRINBERG, M.; STOLF, N. A. G.; VERGINELLI, G.; JATENE, A. D. - Late follow-up of 291 patients who underwent heart valve replacement with mechanical valves. Rev. Bras. Cir. Cardiovasc., 10 (1): 50-55, 1995.

ABSTRACT: Between January 1980 and December 1993, 291 patients underwent valve replacement with mechanical valves in the Heart Institute of HCFMUSP. One hundred and eighty seven (64.3\%) patients were male, aged between 2 months and 78 years (average $38.3+/-18.5$ ). The ethiology was rheumatic in 132 patients $(45.4 \%)$. Were inserted 315 mechanical valves, 201 aortic, 77 mitral, 15 double aortic-mitral, 2 tricuspid, 1 double mitral-tricuspid, and 1 triple mitral-aortic-tricuspid. There were associated procedures in 164 patients $(56.4 \%)$, the most frequent was replacement of ascending aorta in $49(16.8 \%)$. One hundred and forty one patients $(48.4 \%)$ underwent previous heart valve surgery. Follow-up data are reported concerning functional status (NYHA) and valve related complications. Early mortality rate was $12.4 \%$ ( 36 patients). Followup totaled 10078 months/patient (159 patients), with a mean follow-up period of 40.6 months. The linearized rates of thromboembolism, hemorrhage related to antithrombotic therapy, late death, endocarditis, paravalvular leak and hemolysis were $1.33 \%, 0.95 \%, 1.9 \%, 0.19 \%, 0.57 \%$ and $0.57 \%$ patients/year, respectively. Fourteen year survival rate was $63.8 \%$. Eighty two percent of the patients were in functional class $I$ in the postoperative period. It was possible to conclude that the patients submitted to mechanical valve replacement had a satisfactory clinical evolution.

DESCRIPTORS: Heart valves prostheses, surgery, late follow-up. Heart valves, surgery.

\section{REFERÊNCIAS BIBLIOGRÁFICAS}

1 ANDERSON, R. P.; BONCHEK, L. I.; GRUNKEMEIER, G. L.; LAMBERT, L. E.; STARR, A. - The analysis and presentation of surgical results by actuarial methods. J. Surg. Res., 16: 224-230, 1974.

2 ARAMENDI, J. I.; CASTELLANOS, E.; SERRANO, A.; MADIEDO, J. B.; DeSALAZAR, A. O.; AGOSTI, J. A comparative study of the St. Jude Medical valve and Bjork-Shiley convexo-concave prosthesis in isolated valve replacement. J. Cardiovasc. Surg., 32: $557-563,1991$.

3 CARBOMEDICS - The Clinical Report, 6: 1-7, 1994.

4 COSELLI, J. S. \& CRAWFORD, S. - Composite valvegraft replacement of aortic root using separate Dacron tube for coronary artery reattachment. Ann. Thorac. Surg., 47: 558-565, 1989.

5 CZER, L. S. C.; CHAUX, A.; MATLOFF, J. M.; DeROBERTIS, M. A.; NESSIM, S. A.; SCARLATA, D. - Ten-year experience with the St. Jude Medical valve for primary valve replacement. J. Thorac. Cardiovasc. Surg., 100: 44-55, 1990.

6 DeLUCA, L.; VITALE, N.; GIANOLLO, B.; CAFARELLA, G.; PIAZZA, L.; COTRUFO, M. - Midterm follow-up after heart valve replacement with Carbomedics bileaflet prostheses. J. Thorac. Cardiovasc. Surg., 106: 1158-1165, 1993.

7 GREY, D. P.; OTT, D. A.; COOLEY, D. A. - Surgical treatment of aneurysm of the ascending aorta with aortic insufficiency: a selective approach. J. Thorac. Cardiovasc. Surg., 86: 864-877, 1983.

8 JEGADEN, O.; LLOJEH, K.; MONTAGNA, P.; ROSSI, P.; ADELEINE, P.; DELAYE, J. - Resultats éloignés des remplancements valvulaires aortiques isolés par protèse de Björk-Shiley: a propos de 596 cas. Arch. Mal. Coeur, 84: 47-54, 1991.
9

McKAY, C. R. - Prosthetic heart valve thrombosis: what can be done with regard to treatment. Circulation, 87: 294-296, 1993.

10 MORITZ, A.; KLEPETKO, W.; RODLER, S.; FOGER, A.; SCHREINER, W.; GRABENWOGER, F. - Sixyear follow-up after heart valve replacement with the Edwards Duromedics bileaflet prosthesis. Eur. J. Cardio - Thorac Surg., 7: 84-90, 1993.

11 NAIR, C. K.; MOHIUDDIN, S. M.; HILLEMAN, D. E.; SCHULTZ, R.; BAILEY Jr., R. T.; COOK, C. T. - Ten year results with the St. Jude Medical Prosthesis. Am. J. Cardiol., 65: 217-225, 1990.

12 ORSZULAK, T. A.; SCHAFF, H. V.; DeSMET, J. M.; DANIELSON, G. K.; PLUTH, J. R.; PUGA, F. L. Late results of valve replacement with the Björk Shiley valve (1973 to 1982). J. Thorac. Cardiovasc. Surg., 105: 302-312, 1993.

13 RABAGO, G.; CORBI, P.; TEDY, G.; NATAF, P.; FONTANEL, M.; CABROL, C. - Five-year experience with the Medtronic Hall prosthesis in isolated aortic valve replacement. J. Card. Surg., 8: 85-88, 1993.

14 SILBER, H.; KHAN, S. S.; MATLOFF, J. M.; CHAUX, A.; DeROBERTIS, M.; GRAY, R. - The St. Jude valve: thrombolysis as the first line of therapy for cardiac valve thrombosis. Circulation, 87: 30-37, 1993.

15 SVENSSON, L. G.; CRAWFORD, S.; HESS, K.; COSELLI, J. S.; SAFI, H. J.- Composite valve graft replacement of the proximal aorta: comparison of techniques in 348 patients. Ann. Thorac. Surg, 54: 427-439, 1992.

16 TEPLY, J. F.; GRUNKEMEIER, G. L.; SUTHERLAND, H. D.; LAMBERT, L. E.; JOHNSON, V. A.; STARR, A. - The ultimate prognosis after valve replacement: an assessment at twenty years. Ann. Thorac. Surg. 32: $111-119,1981$ 